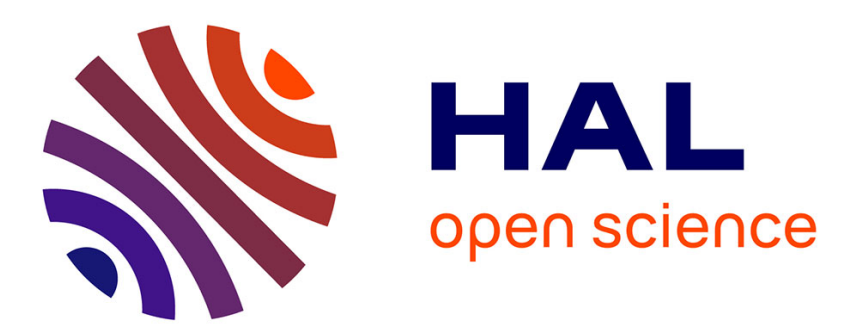

\title{
Diffusiophoretic design of self-spinning microgears from colloidal microswimmers
}

\author{
Antoine Aubret, Jérémie Palacci
}

\section{To cite this version:}

Antoine Aubret, Jérémie Palacci. Diffusiophoretic design of self-spinning microgears from colloidal microswimmers. Soft Matter, 2018, 14 (47), pp.9577 - 9588. 10.1039/c8sm01760c . hal-03279178

\section{HAL Id: hal-03279178 \\ https://hal.science/hal-03279178}

Submitted on 6 Jul 2021

HAL is a multi-disciplinary open access archive for the deposit and dissemination of scientific research documents, whether they are published or not. The documents may come from teaching and research institutions in France or abroad, or from public or private research centers.
L'archive ouverte pluridisciplinaire HAL, est destinée au dépôt et à la diffusion de documents scientifiques de niveau recherche, publiés ou non, émanant des établissements d'enseignement et de recherche français ou étrangers, des laboratoires publics ou privés. 


\section{Accepted Manuscript}

This article can be cited before page numbers have been issued, to do this please use: A. Aubret and J.

Palacci, Soft Matter, 2018, DOI: 10.1039/C8SM01760C.

\section{Soft Matter}

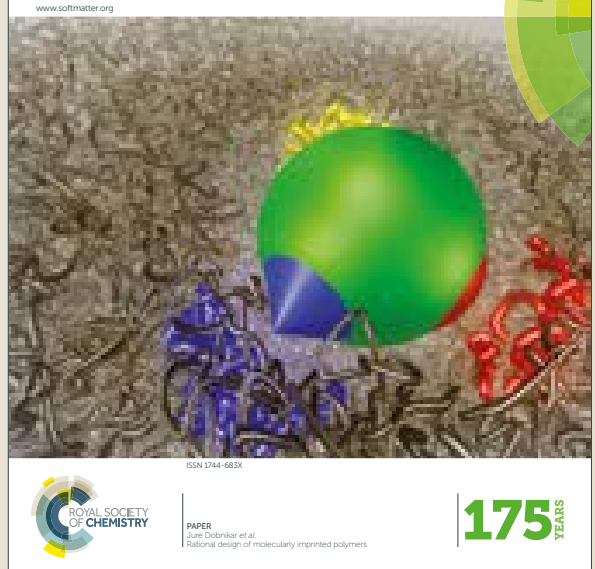

This is an Accepted Manuscript, which has been through the Royal Society of Chemistry peer review process and has been accepted for publication.

Accepted Manuscripts are published online shortly after acceptance, before technical editing, formatting and proof reading. Using this free service, authors can make their results available to the community, in citable form, before we publish the edited article. We will replace this Accepted Manuscript with the edited and formatted Advance Article as soon as it is available.

You can find more information about Accepted Manuscripts in the author guidelines.

Please note that technical editing may introduce minor changes to the text and/or graphics, which may alter content. The journal's standard Terms \& Conditions and the ethical guidelines, outlined in our author and reviewer resource centre, still apply. In no event shall the Royal Society of Chemistry be held responsible for any errors or omissions in this Accepted Manuscript or any consequences arising from the use of any information it contains. 


\title{
Journal Name
}

\section{ARTICLE TYPE}

Cite this: DOI: $10.1039 / x x x x x x x x x x$

\section{Diffusiophoretic design of self-spinning microgears from colloidal microswimmers ${ }^{\dagger}$}

\author{
Antoine Aubret, ${ }^{* a}$ and Jérémie Palacci ${ }^{a}$
}

Received Date

Accepted Date

DOI: $10.1039 / x x x x x x x x x x$

www.rsc.org/journalname

\begin{abstract}
The development of strategies to assemble microscopic machines from dissipative building blocks are essential on the route to novel active materials. We recently demonstrated the hierarchical self-assembly of phoretic microswimmers into self-spinning microgears and their synchronization by diffusiophoretic interactions [Aubret et al., Nature Physics, 2018]. In this paper, we adopt a pedagogical approach and expose our strategy to control self-assembly and build machines using phoretic phenomena. We notably introduce Highly Inclined Laminated Optical sheets microscopy (HILO) to image and characterize anisotropic and dynamic diffusiophoretic interactions, which cannot be performed by conventional fluorescence microscopy. The dynamics of a (haematite) photocalytic material immersed in (hydrogen peroxide) fuel under various illumination patterns is first described and quantitatively rationalized by a model of diffusiophoresis, the migration of a colloidal particle in a concentration gradient. It is further exploited to design phototactic microswimmers that direct towards the high intensity of light, as a result of the reorientation of the haematite in a light gradient. We finally show the assembly of self-spinning microgears from colloidal microswimmers and carefully characterize the interactions using HILO techniques. The results are compared with analytical and numerical predictions and agree quantitatively, stressing the important role played by concentration gradients induced by chemical activity to control and design interactions. Because the approach described hereby is generic, this works paves the way for the rational design of machines by controlling phoretic phenomena.
\end{abstract}

\section{Introduction}

The self-assembly of individual building blocks into functional units is a fundamental goal of science and engineering. Progress has been made in the assembly of colloidal particles into isotropic, chiral, and directional equilibrium structures ${ }^{1}$ using DNA origami ${ }^{2,3}$, entropic forces ${ }^{4}$, anistropic particles ${ }^{5,6}$, or field induced interactions ${ }^{7-10}$. The resulting equilibrium structures are static in the absence of an external force, for example optical tweezers ${ }^{11}$ or magnetic field ${ }^{7,12,13}$.

Dissipative self-assembly from building blocks that consume energy alternatively allows architectures that are dynamical and reconfigurable ${ }^{14,15}$, a desirable feature of advanced materials. Man-made realization of dynamical self-assembly in synthetic systems were first limited to macroscopic systems ${ }^{16,17}$ but recent progress in colloidal science have made available self-propelled colloids (or microswimmers) ${ }^{18-21}$ and allowed overcoming this limitation. They notably forged the rise of Active Matter as a

${ }^{a}$ Department of Physics, University of California, San Diego, USA; E-mail: aaubret@ucsd.edu

$\dagger$ Electronic Supplementary Information (ESI) available: [details of any supplementary information available should be included here]. See DOI: $10.1039 /$ CXsm00000x/ field and permitted the exploration of emergent phenomena: dynamic phase transition, clustering, living crystals, or flocking, as examples of self-organization ${ }^{14,22}$ as well as the field-driven phase-behaviors of collective assemblies ${ }^{23,24}$. The realization of dynamical structures made from microswimmers is however lagging ${ }^{14,15}$, highlighting the lack of simple design rules for dissipative self-assembly ${ }^{25,26}$. Field-driven approaches have been successful in assembling rotors, chains or clusters of microswimmers actuated by oscillating electric or magnetic fields ${ }^{23,24,27,28}$. While the dynamics can be changed by tuning the frequency and amplitude of the fields, the control of the structure at the individual level is lacking. Templated assembly tackles the issue by guiding the assembly using boundaries to direct self-propelled particles $^{29,30}$ and rectify motion ${ }^{29-35}$, however at the expense of limited flexibility and control from preliminary microfabrication requirements. An alternate approach uses light patterns to encode information ${ }^{36-40,40-44}$, in place of microscopic templates. It is highly flexible as the the spatiotemporal modulation of illumination controls particles' velocity with micrometric resolution and the convenience of the flick of a switch ${ }^{45}$.

In this paper, we show that light patterns together with photocatalytic particles allow controlling the phenomenon of diffu- 
siophoresis, the motion of a colloidal particle along a gradient of chemicals ${ }^{46,47,47-50}$, to design anisotropic and dynamic diffusiophoretic interactions. This leads to the robust and faithful assembly of phototactic microswimers into autonomous selfspinning microgears. We adopt a pedagogical, step by step, presentation, starting from the characterization of a photocatalytic haematite component until the hierarchical design of a spinning micromachine. We first engineer a phototactic microswimmers $39,40,42-44,51-53$. Next, we harness their response to light gradients to trigger their self-assembly in a controllable and robust manner. Following adequate light signal, the microswimmers autonomously form chiral rotors, which sustain under uniform illumination. The rotor constitutes a mesomachine and creates a dynamical and anisotropic cloud of chemicals that induce a repulsive diffusiophoretic interaction, which we characterize using Highly Inclined Laminated Optical sheets microscopy (HILO). The results agree with analytical and numerical predictions of a simple model of a rotor forming a hexapolar sink of fuel. Our results demonstrate the hierarchical construction of machines from machines by taking advantage of phoretic phenomena and light to direct self-assembly.

\section{Response of a photocalytic material, haematite, to light gradients}

\subsection{Haematite, a photocatalytic material}

We harness the photocatalytic properties of a piece of semiconducting haematite iron oxide $\alpha-\mathrm{Fe}_{2} \mathrm{O}_{3}$ immersed in a solution of hydrogen peroxide $\mathrm{H}_{2} \mathrm{O}_{2}$ to transduce chemical energy into mechanical work. A diluted solution of $\mathrm{H}_{2} \mathrm{O}_{2}$ spontanously degrades in presence of a catalyst: $2 \mathrm{H}_{2} \mathrm{O}_{2} \rightarrow 2 \mathrm{H}_{2} \mathrm{O}+\mathrm{O}_{2}$. In a nutshell, haematite creates electron-hole pairs following illumination by UV-blue light. While spontaneous recombination usually occurs over a few nanoseconds, holes and electrons bounded to the surface allow redox processes to occur, catalyzing the $\mathrm{H}_{2} \mathrm{O}_{2}$ decomposition. The overall reaction scheme is complex, involving many intermediate species such as hydroxide radicals ${ }^{54}$, but eventually leads to $\mathrm{O}_{2}$ and $\mathrm{H}_{2} \mathrm{O}$ production. The release of chemical energy from the degradation of $\mathrm{H}_{2} \mathrm{O}_{2}$ is exploited to inject energy locally and maintain the system away from equilibrium. We explore the response of haematite micrometric particles (size $\sim 0.5-1 \mu \mathrm{m}$ ) under various illumination patterns and immersed in a solution of fuel obtained by dilution of $\mathrm{H}_{2} \mathrm{O}_{2}$ in DI water (Millipore, 18.2M $\Omega$ ) and made slightly acidic, $\mathrm{pH} \sim 6-7$, by dissolved carbon dioxide from the atmosphere (Fig.1A, see Methods for sample preparation).

\subsection{Tethered haematite in uniform illumination}

We first study a single haematite particle under uniform illumination and examine the diffusiophoretic behavior of colloidal beads in presence of the subsequent concentration gradients. We tether a haematite particle on the surface of a capillary and introduce a solution containing $20 \mathrm{~nm}$ nile red fluorescent beads, which diffusiophoretic response will probe the underlying concentration gradient. In standard epifluorescence microscopy, the complete sample is excited, resulting in strong background fluorescence, hin-
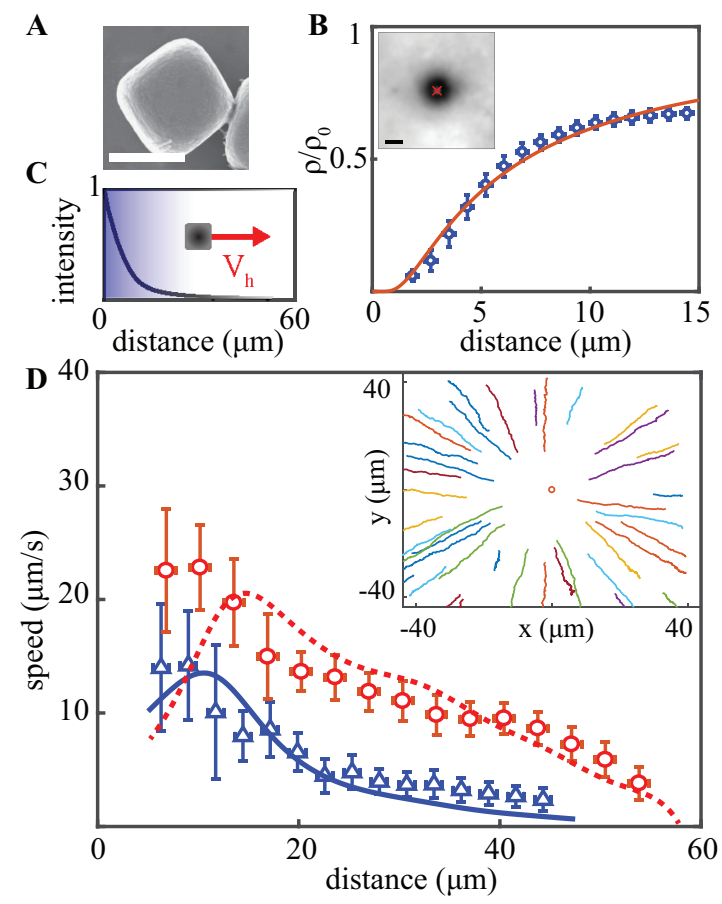

Fig. 1 Response of haematite under illumination. A) SEM image of a haematite particle. Scale bar is $500 \mathrm{~nm}$. B) Isotropic phoretic repulsion of $20 \mathrm{~nm}$ fluorescent beads around a haematite particle tethered on the surface of a capillary, under uniform blue $(\lambda=395 \mathrm{~nm})$ excitation. The density of fluorescent beads (blue circles) decays when approaching the haematite particle, and follows eq.(2) (red line), with $\alpha=64 \mu \mathrm{m}^{3} / \mathrm{s}$. Inset: repulsion of FB in the XY plane. The red cross locates the haematite particle (scale bar $5 \mu \mathrm{m}$ ). C) Isotropic intensity profile generated with a slightly defocused blue laser spot $(\lambda=404 \mathrm{~nm})$, after azimuthal averaging, used for analyzing the response of haematite in a gradient of light. An asymmetric reaction rate occurs at the surface of the haematite, triggering its motion at speed $\mathbf{V}_{\mathrm{h}}$ by self-diffusiophoresis. D) Recorded speeds of haematite particles in the intensity gradient shown in $\mathrm{C}$ ), at excitation power of $\mathrm{P}=0.16 \mathrm{~mW}$ (blue triangles) and $\mathrm{P}=1 \mathrm{~mW}$ (red circles). The velocity is adjusted with eq.(3) (solid blue line and dashed red line) from the measured intensity profile and using constant saturation intensity (see main text). Inset : trajectories of individual haematite particles, isotropically repelled from the laser spot (red dot), at $\mathrm{P}=1 \mathrm{~mW}$.

dering quantitative measurement of the phoretic interactions (see Methods and Fig.6D). To overcome this problem, we use Highly Inclined and Laminated Optical sheet microscopy technique ${ }^{55-57}$ (HILO, or near-TIRF) that narrows the observation space to a thin slice near the bottom of the capillary, where the particles sit (see Methods). In brief, the technique involves a setup similar to Total Internal Reflection Fluorescence microscopy (TIRF), with the beam forming an angle slightly smaller than the critical angle. It results in a highly inclined beam that excites only a few microns above the substrate, and allows us to analyze the density of the fluorescent beads around a haematite. The rapid diffusion of the nanometric colloidal tracer provides a quick equilibration over the vertical space and produces an measurement integrated over the illuminated slice. In the absence of blue illumination or $\mathrm{H}_{2} \mathrm{O}_{2}$ fuel, the concentration of fluorescent beads is uniform. An exclusion zone develops near the haematite particle when it is photo-activated by blue light: the beads are phoretically repelled and drift away in the isotropic gradient induced by 
the photocatalytic activity of haematite (Fig.1B). The concentration of $\mathrm{H}_{2} \mathrm{O}_{2}$, c, obeys the Laplace equation $\Delta c=0$ with a sink placed at the position of the haematite, $r=0$ giving $c(r) \propto 1 / r$. A colloidal particle placed in a chemical gradient $\nabla c^{\prime}$ exhibits a diffusiophoretic migration with a velocity $v_{D P} \propto \nabla c^{\prime 46}$. The details of the phoretic mechanism at the origin at the migration are complex as the degradation of $\mathrm{H}_{2} \mathrm{O}_{2}$ involves many (intermediate) reaction products with unknown solvent/particle interaction. Exothermic steps in the degradation could also add up a thermophoretic component to the migration, without changing the scaling of the interaction (see below). Moreover, and as discussed in previous work ${ }^{38,45}$, the direction of the migration can be reversed by shifting the $\mathrm{pH}$ of the solution to basic. We focus in this manuscript on the characterization of the effective and repulsive interaction induced by the consumption of $\mathrm{H}_{2} \mathrm{O}_{2}$ at slightly acidic $\mathrm{pH} \sim 6-7$ and its use to design and control assembly. . We write $v_{D P}=D_{\mathrm{DP}} \nabla c$, with $D_{\mathrm{DP}}$ the phoretic mobility, which depends on the particle-solvent interaction. For the tethered haematite, we predict a phoretic repulsion for a fluorescent bead at distance $r$ with velocity $v_{\mathrm{DP}}=\alpha / r^{2}$, where $\alpha$ is a parameter describing the effective phoretic repulsion, dependent on reaction rates ${ }^{50}$. The flux of particles of density $\rho$ writes as drifting phoretically and subjected to brownian motion:

$$
\mathbf{J}=-D_{\mathrm{c}} \nabla \rho+\mathbf{v}_{\mathrm{DP}} \rho
$$

with $\rho$ the particles density, $D_{\mathrm{c}}=18 \mu \mathrm{m}^{2} / \mathrm{s}$ the thermal diffusion coefficient of the beads. At steady state, $\mathbf{J}=0$, and integration of eq.(1), leads to the radial intensity profile in the (XY) plane:

$$
\rho=\rho_{0} e^{-\alpha /\left(D_{\mathrm{c}} r\right)}
$$

This is analogous to an effective Boltzmann distribution of the beads in the repulsive potential $U(r) \propto c(r)=\alpha / r$. We use eq. (2) to adjust our data using $\rho_{0}$ and $\alpha$ as fitting parameters, and find an excellent agreement for $\alpha=64 \pm 10 \mu \mathrm{m}^{3} / \mathrm{s}$ (Fig.1B). A haematite particle acts as a sink of $\mathrm{H}_{2} \mathrm{O}_{2}$ and repels the surrounding particles by diffusiophoresis.

\subsection{Untethered haematite under light gradients}

Untethered haematite in uniform light shows random motion without apparent persistent propulsion. Following, we study the behavior of untethered haematite particles in light gradients (see supplementary movie S1) by using a slightly defocused laser spot projected on the bottom surface of the sample (Fig.1C, $\lambda=404$ $\mathrm{nm}$, see Methods). We initially locate haematite particles in the sample, position the laser spot in their vicinity and record the motion of the particles, isotropically repelled away from the laser spot. We repeat the experiment over several dozens of individual haematite particles and extract the evolution of the speed in the radial direction. The procedure is repeated for 5 different intensities, ranging from $0.03 \mathrm{~mW}$ to $1 \mathrm{~mW}$ (peak intensities of $\approx 0.06$ to $2 \mu \mathrm{W} / \mu \mathrm{m}^{2}$ ). The positions are averaged over $1 \mathrm{~s}$ and the speed is calculated as a function of the position for each particle. We average the data over $\approx 30$ haematite particles for each intensity profile, measured and quantified independently (Fig.1C, see
Methods). The particles travel radially along the light gradients, migrating towards lower intensities. The speed decreases with increasing distance from the laser, as presented in Fig.1D.

As the reaction rate of decomposition of $\mathrm{H}_{2} \mathrm{O}_{2}$ depends on light intensity, haematite particles in light gradients present spatial gradients of reaction rates on their surface inducing a subsequent phoretic velocity $V \propto \nabla v^{50}$ (Fig.1C). The photoactivity of haematite is proportional to the absorbed intensity $I_{\mathrm{abs}}$, which saturates at high intensity $I_{\mathrm{sat}}^{\mathrm{h}}: I_{\mathrm{abs}} \propto I /\left(1+I / I_{\mathrm{sat}}^{\mathrm{h}}\right)$. We subsequently obtain the phoretic velocity for the untethered haematite particle:

$$
V_{\mathrm{h}}(I)=\chi \frac{\nabla I}{\left(1+I / I_{\mathrm{sat}}^{\mathrm{h}}\right)^{2}}
$$

where $\chi$ and $I_{\text {sat }}^{\mathrm{h}}$ characterize the phoretic response of the particle and its absorption properties, respectively. The saturation intensity $I_{\text {sat }}^{\mathrm{h}}$ is a material property, independent of the illumination intensity as the experiment is performed in a reaction-limited regime $^{58}$. By measuring the intensity profile $I(r)$, we compare our model with the measured velocities and obtain a good agreement with eq. (3) for $I_{\text {sat }}^{\mathrm{h}} \sim 75 \mathrm{nW} / \mu \mathrm{m}^{2}$ (see Fig.1D). The agreement holds over 2 orders of magnitude for the illumination, ranging from 0.03 to $1 \mathrm{~mW}$ without altering $I_{\text {sat }}^{\mathrm{h}}$.

\section{Design of a phototactic swimmer}

We take advantage of the response of haematite to light gradients to design phototactic Janus microswimmers, consisting of an inert polymer bead (3-(trimethoxysilyl)propyl methacrylate, TPM, Sigma Aldrich), from which a haematite particle is extruded (see Fig.2A) ${ }^{18,19}$, forming a swimmer with fore-aft asymmetry and typical length $l=1.8 \mu \mathrm{m}$. We introduce the swimmers in a capillary containing a $6 \%$ solution of hydrogen peroxide $(\mathrm{pH} \sim 6-7)$. The swimmers sediment close to the surface and the observations are performed in this plane. In the absence of illumination, the particles undergo thermal motion and do not self-propel.

\subsection{Microswimmer in uniform light}

We analyze the behavior of the swimmers under uniform light and record their trajectories for various intensities (Fig.2B). The swimmers self-propel with the bead heading, in line with our previous observations that beads migrate toward the high concentration of $\mathrm{H}_{2} \mathrm{O}_{2}$ by diffusiophoresis ${ }^{20,46,47,47-50}$ (Fig.1B). The swimmers exhibit persistent random walk : they travel in straight lines before thermal fluctuations randomize the direction of their motion on a timescale given by the rotational diffusion time $\tau_{r}$. The Mean Square Displacement (MSD) $(\Delta L)^{2}$ at time $\Delta t$ is extracted as 49,59 :

$$
(\Delta L)^{2}=4 D_{s} \Delta t+\frac{V_{s}^{2} \tau_{r}^{2}}{2}\left[\frac{2 \Delta t}{\tau_{r}}+\mathrm{e}^{-2 \Delta t / \tau_{r}}-1\right]
$$

with $D_{s}$ the diffusion coefficient of the swimmers at equilibrium (no illumination), and $V_{s}$ their speed. We measure $D_{s}$ from equilibrium experiments with 289 swimmers, and find a value of $D_{s}=0.3 \pm 0.1 \mu \mathrm{m}^{2} / \mathrm{s}$. Following, we extract the rotational diffusion $D_{r}=3 D_{s} / 4 R^{2}=0.3 \pm 0.1 \mathrm{rad}^{2} / \mathrm{s}$, and the corresponding rotational diffusion time $\tau_{r}=1 / D_{r} \approx 3 \mathrm{~s}$. We adjust the MSD 

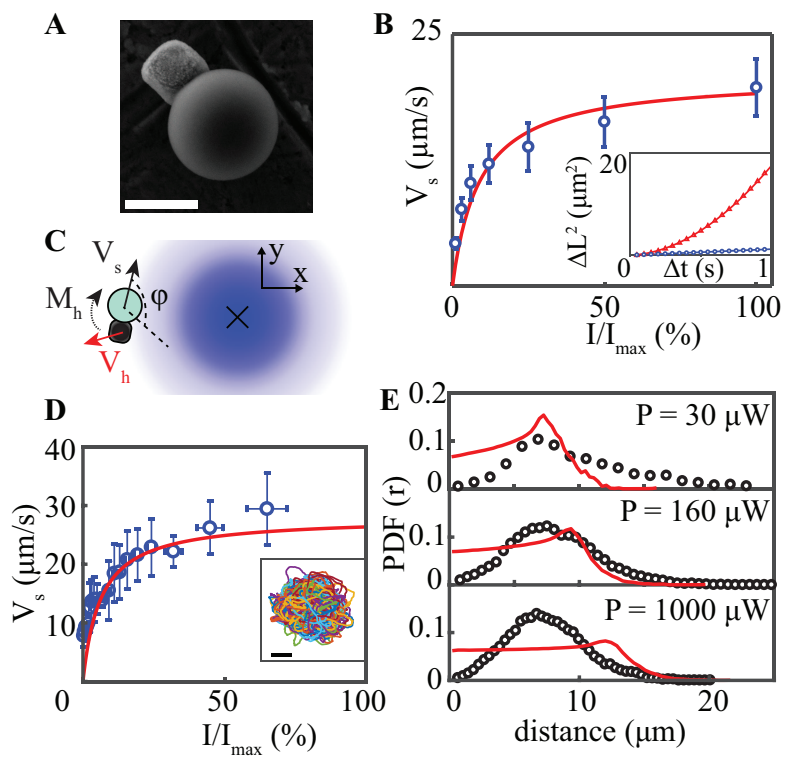

Fig. 2 A phototactic microswimmer. A) SEM image of the phototactic swimmer, which consists of a haematite particle extruded from a colloidal bead. Scale bar is $1 \mu \mathrm{m}$. B) The swimmer exhibits persistent random walk under homogeneous illumination with a velocity (blue circles) tuned by the light intensity $\left(I_{\max }=550 \mathrm{nW} / \mu \mathrm{m}^{2}\right)$. The red line is a fit using eq.(5), and $I_{\text {sat }}=50 \mathrm{nW} / \mu \mathrm{m}^{2}$. Inset shows the Mean Square Displacement $(\Delta L)^{2}$ of swimmers at equilibrium (no illumination, blue circles) and under illumination (red triangles), at $I / I_{\max }=10^{-2}$. Solid lines are fits with eq.(4). For $I=0$, we get $D_{s}=0.3 \pm 0.1 \mu \mathrm{m}^{2} / \mathrm{s}$. C) Scheme of the phototactic mechanism. As the haematite is phoretically repelled from higher intensities of light, it reorients the swimmer along the gradient, inducing an effective torque $\mathbf{M}_{\mathbf{h}}$. The blue spot represents the laser used in the experiment and the notations are the ones from main text. D) Evolution of the speed $V_{s}(I)$ of individual swimmers under the same excitation profile and intensities as for the haematite in section $2,\left(I_{\max }=1200\right.$ $\left.\mathrm{nW} / \mu \mathrm{m}^{2}\right)$. The solid line follows eq.(5), with $V_{\text {sat }}=24 \mu \mathrm{m} / \mathrm{s}$ and $I_{\text {sat }}=75$ $\mathrm{nW} / \mu \mathrm{m}^{2}$. Inset shows the trajectories of the swimmers around the laser spot. Scale bar is $5 \mu \mathrm{m}$. E) Density of Probability of Presence of the swimmers (PDF) at distance $r$ from the center for 3 different excitation powers (black circles). It shows a reasonable agreement with our simulations of ABP, notably the range of the trajectories, without any adjustable parameter (solid red lines, see main text).

curves with eq.(4), using $V_{s}$ as only fitting parameter and obtain the dependency of the phoretic speed on the intensity (Fig.2B). The velocity increases rapidly at low intensities, and saturates at higher intensities:

$$
V_{s}(I)=V_{\text {sat }} \frac{I}{1+I / I_{\text {sat }}},
$$

where $I_{\text {sat }}$ and $V_{\text {sat }}$ are the illumination and speed at saturation, respectively. A good agreement is found with the experiment for $V_{\text {sat }}=21 \mu \mathrm{m} / \mathrm{s}$ and $I_{\text {sat }} \sim 50 \mathrm{nW} / \mu \mathrm{m}^{2}$ at $\lambda=395 \mathrm{~nm}$ (see Methods), and consistent with the saturation value for the illumination extracted for the haematite alone, $I_{\text {sat }}^{\mathrm{h}} \sim 75 \mathrm{nW} / \mu \mathrm{m}^{2}$, at a slightly different wavelength $\lambda=404 \mathrm{~nm}$.

\subsection{Microswimmers in two-dimensional light gradients}

The behavior of the swimmer qualitatively changes in nonuniform light, as they migrate towards high intensities for any given intensity profile (Fig.2C and supplementary movie S2), a dynamics akin to the phenomenon of phototaxis ${ }^{39,40,42-44,51-53}$. In order to quantify the phototactic response, we record the motion of the microswimmers at the bottom surface of the cell using the same excitation profiles (intensity and spatial distribution) that we used for the haematite alone in section 2 and obtained by shining a laser spot in the absence of a uniform background illumination. The swimmers travel radially, cross near the center of the laser spot and reverse direction to reorient along the light gradient and towards the center, a dynamics that appears similar to previously reported thermophoretic particles oriented by scattering forces using a high intensity laser spot ${ }^{60}$. The origin of the present phenomenon is however different as the reorientation results from the phoretic migration of the haematite component towards the low intensity of light (see Fig.2C). In order to validate this scenario, we compare the observed trajectories with simulated trajectories of a model Active Brownian Particle $(A B P)^{14,22}$, where all the parameters are determined from independent measurements performed on individual haematite particles and swimmers. The motion of phoretic microswimmers is torque and force free ${ }^{46}$ but the equations of motion of a microswimmer in the overdamped regime can be rewritten in terms of effective forces and effective torques ${ }^{61}$. We first reconstruct the trajectories of the swimmers and extract their speed $V_{S}(r)$, at distance $r$ from the laser spot and intensity dependence $V_{S}(I)$ under the specific excitation profile (Fig.2D). The experimental velocity of a microswimmer follows eq.(5), with $I_{\text {sat }} \sim 75 \mathrm{nW} / \mu \mathrm{m}^{2}$, and $V_{\text {sat }}=24 \mu \mathrm{m} / \mathrm{s}$. We consider the instantaneous velocity of the swimmer depending only on the local value of the intensity: $V_{s}=V_{s}(x, y)$. A swimmer at time $t$ is situated at the position $(x, y)$, with orientation $\mathbf{u}_{\varphi}$ forming the angle $\varphi$ with the x-axis, in the reference frame centered at $(0,0)$ on the laser spot (Fig.2C). We describe the reorientation of the swimmer by an effective torque $\mathbf{M}_{\mathbf{h}}$ induced by the migration of the haematite component:

$$
\mathbf{M}_{\mathbf{h}}=-R \gamma \mathbf{u}_{\varphi} \times \mathbf{V}_{\mathbf{h}}
$$

where $\gamma=6 \pi R \eta$ is the viscous drag coefficient ( $\eta$ the dynamic viscosity of the water). The velocity of the haematite alone is radial and writes $\mathbf{V}_{\mathrm{h}}=V_{\mathrm{h}} \mathbf{u}_{\mathbf{r}}$, and is given by eq.(3). The other parameters, $\chi, I_{\text {sat }}^{\mathrm{h}}, I(r)$ and $\nabla I(r)$, have been previously extracted from independent experiments thus characterizing $\mathbf{M}_{\mathbf{h}}$ unequivocally from measurements on haematite particles only.

Following previous studies ${ }^{60-62}$, we write the Langevin equations for the motion of the swimmer in the $(X Y)$ plane as:

$$
\begin{aligned}
& \frac{\mathrm{d} x}{\mathrm{~d} t}=V_{s}(x, y) \mathbf{u}_{\mathbf{x}} \cdot \mathbf{u}_{\varphi}+\sqrt{2 D_{s}} \zeta_{x}(t) \\
& \frac{\mathrm{d} y}{\mathrm{~d} t}=V_{s}(x, y) \mathbf{u}_{\mathbf{y}} \cdot \mathbf{u}_{\varphi}+\sqrt{2 D_{s}} \zeta_{y}(t) \\
& \frac{\mathrm{d} \varphi}{\mathrm{d} t}=\frac{M_{h}}{\gamma R^{2}}+\sqrt{2 D_{r}} \zeta_{\varphi}(t)
\end{aligned}
$$

with $\zeta$ a noise term with zero mean and variance, such that $<\zeta(\tau) \zeta(\tau+t)>=\delta(t)$. Using the values we measured for $\chi$, $I_{\mathrm{sat}}=I_{\mathrm{sat}}^{\mathrm{h}}=75 \mathrm{nW} / \mu \mathrm{m}^{2}, V_{\mathrm{sat}}=24 \mu \mathrm{m} / \mathrm{s}$, and the experimental 
intensity profile (see Fig.1D), we simulate the trajectories of the swimmers for each investigated intensities.

We plot the radial density of probability of presence of the swimmer in Fig.2E, and find a good qualitative agreement with the experimental data (Fig.2E). We notably reproduce the spatial extension of the trajectories around the laser spot. The absence of free parameter in eq.(7) ascertains the role of the phoretic migration of haematite in the orientation process. Discrepancies between the simulations and the experiment arise at short distance $r$, where the focus of the laser is intense $\left(I \sim \mu \mathrm{W} / \mu \mathrm{m}^{2}\right)$. Scattering of light by haematite, repelled from the center by radiative pressure and potential exothermic $\mathrm{H}_{2} \mathrm{O}_{2}$ decomposition at the intense focus of the laser could both lead to chemical gradients and subsequent repulsion away from the laser spot. We did not attempt to capture those effects in our model as it is implied by the high intensity used in the characterization experiments and would not illuminate any aspect of the phoretic dynamics of our system. The presented strategy constitutes a generic rule for the design of autonomous particles directed by light gradients.

\subsection{Effects of vertical light gradients on the microswimmers}

As haematite scatters in blue, it leads to shadowing ${ }^{52}$ that sets a vertical gradient of light along the direction of the laser. The haematite migrates along the light gradient to the region of low intensity (see Section 3.2). If the laser is shone from above, the swimmer reorients with the haematite under and leaves the surface, leading to a 3D motion in the bulk of the sample. If the laser is shone from below, the haematite is phoretically lifted and the composite particle tends to flip when approaching the laser focus, swimming downwards and pushing against the bottom surface. Alternate reorientation mechanisms near surfaces have been reported for catalytic microswimmers, as a result of a subtle interplay between hydrodynamics and modifications of the concentration gradients by impermeable walls ${ }^{63-65}$. Their contribution to the flipping mechanism cannot be ruled out, but the dependence of the observed motion on the position of the laser indicates that, in our experiment, phoresis is an essential component to the observed flipping of the microswimmer. We further use this mechanism to assemble self-spinning microgears (movie S3).

\section{Guided self-assembly of self-spinning mi- crogears}

We initiate the experiment with a dilute sample, $\Phi_{s} \sim 10^{-3}$ part $/ \mu \mathrm{m}^{2}$, of phototactic microswimmers under uniform illumination. They travel along the substrate and the density is too low to observe formation of clusters by Motility Induced Phase Separation (MIPS) ${ }^{66,67}$. Following, we superimpose the spot of a focused laser laser to direct the particles by phototaxis. Responding to the light gradient, the swimmers direct their motion towards the laser spot, locally increasing the density. They collide and one swimmer flips and sits vertically, facing the substrate and pushing against the wall. This results in a short-range pumping flow as discussed in refs. ${ }^{65,68}$. It attracts and traps neighboring swimmers into a peripheral crown to form a self-spinning microgear (movie S3). It is composed of 7 particles; one central swimmer with the haematite pointing up, surrounded by 6 close-packed particles facing the central one. Each haematite component in the structure constitutes a chemical sink of $\mathrm{H}_{2} \mathrm{O}_{2}$ and interacts through phoresis with the neighboring swimmers (see section 2). The peripheral swimmers collectively orient in the same direction, setting chirality and rotation of the assembly. Once the structure is formed, the laser can be switched off and the microgear is stable and spins under homogeneous illumination (see Fig.3A).

The reorientation and collective tilt of the peripheral particles that set the chirality of the structure can be understood as a result of the competition between hard sphere interactions and the diffusiophoretic repulsion between the swimmers, induced by their haematite part. This mechanism also rationalizes the absence of a second peripheral layer: the diffusiophoretic repulsion overwhelms the attractive (short-range) hydrodynamic pumping and guarantees the fidelity and robustness of the assembly: additional particles spontaneously detach (movie S3, S4). The importance of phoresis has been recently pointed out for cross-interaction between phoretically propelled particles ${ }^{69-71}$ but hydrodynamic flows could also play a non-negligible role, difficult to estimate from the complex geometry of the system in the near-field and potentially hindered by the near presence of the bottom substrate. Measurements with tracers particles that do not present phoretic mobility (currently unavailable), or the statistics of reversal of chirality could potentially illuminate those aspects of the assembly and nurture further theoretical and experimental studies but are beyond the scope of this manuscript.

\subsection{Rotors dynamics}

We form isolated rotors by superimposing a laser spot to a uniform illumination background, with $P>1 \mathrm{~mW}$, the minimal power of the laser (spot size of $0.5-5 \mu \mathrm{m}$ ) to trigger the selfassembly. Once the rotor is formed, we track the motion of all particles forming the microgear, and reconstruct their individual trajectories. The experiment is repeated for multiple independent rotors and intensities of light to study the dynamics.

The center of mass of individual rotors exhibits a diffusive behavior in uniform light, with diffusion $D_{\mathrm{R}}=0.4 \pm 0.1 \mu \mathrm{m}^{2} / \mathrm{s}$ (Fig.3E). This value is higher than the diffusion constant expected from a passive particle of this size $(R \approx 3 \mu \mathrm{m})$ and arises from active noise. The center of the rotors can be optically trapped with a red laser ( $\lambda=685 \mathrm{~nm}, P \sim 10-100 \mu \mathrm{W})$ without disturbing the system as the absorption of red light is weak and has negligible effect on the photocatalytic activity. It allows suppressing the random motion in the center of mass (Fig.3E-Inset) and focus on the accurate determination of the angular speed of the rotation. It is tuned by the light intensity and reflects the change of translational speed of the swimmer $V_{s}$ with a $\sim \pi / 4$ incline: $\omega R / V_{s} \sim \sqrt{2} / 2$ (Fig.3B). At low rotation rate $(\omega<3 \mathrm{rad} / \mathrm{s})$, thermal fluctuations can flip the direction of the spinning, but the magnitude of the speed of the rotor remains constant (Fig.3C). Further reducing the intensity reduces stability and the rotor breaks into 7 individual microswimmers. At higher speeds ( $\omega>3 \mathrm{rad} / \mathrm{s}$, Fig.3D-Inset), the rotors are robust and the motion is persistent over the dura- 

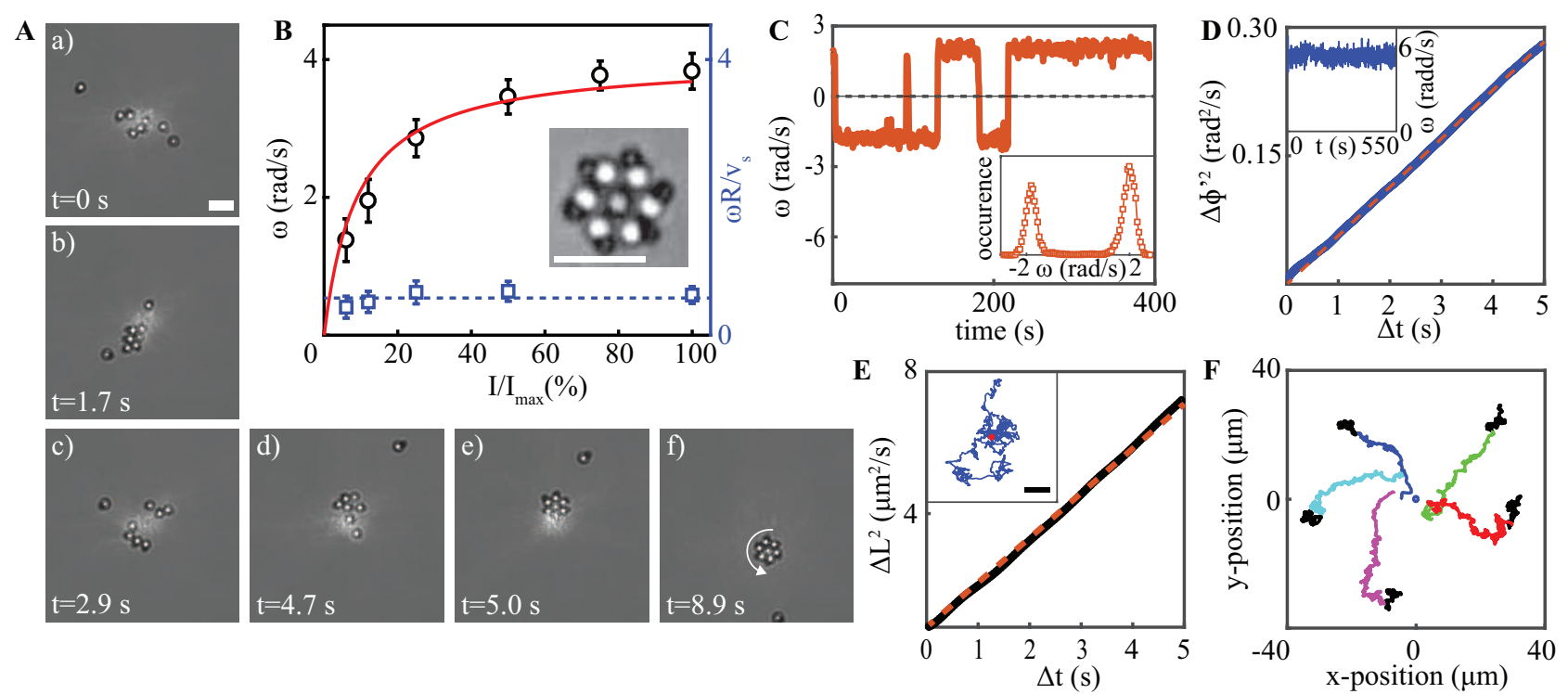

Fig. 3 Formation mechanism and properties of the rotor. A) Snapshots of a movie showing the assembly of a rotor around a single laser spot. In (a) the swimmers travel around the laser spot, until they assemble, (b) and form a transient structure that breaks, (c). The swimmers collide again, (d) and form a stable rotor (e), which is sustained under illumination after removing the gradient (f). B) The angular speed of the rotor is tuned by the light intensity. The solid red line is a fit with eq.(5), using the same saturation intensity as for the swimmers alone. The angular speed reflects the speed of the swimmers (blue squares). Inset: magnified image of a rotor, where the orientation of the swimmers is visible. C) At low angular speeds $(\omega<3$ $\mathrm{rad} / \mathrm{s}$ ), the rotor can flip its direction of rotation. Inset: distribution of speeds for a rotor during a $\sim 10$ min experiment. The two peaks are situated at -1.8 and $1.9 \mathrm{rad} / \mathrm{s}$, showing the symmetry of the dynamics. D) At higher speeds, the motion is persistent and the angular fluctuations can be extracted (blue line). Dashed red line shows a linear fit using eq. (8), and gives $D_{\Phi}=0.03 \pm 0.01 \mathrm{rad}^{2} / \mathrm{s}$. Inset: speed of a fast rotor $(\omega \sim 6 \mathrm{rad} / \mathrm{s})$ over $10 \mathrm{~min}$. E) Experimental MSD (black curve) of free-to-move rotors, and linear fit (red dashed line), giving $D_{\mathrm{R}}=0.4 \pm 0.1 \mu \mathrm{m}^{2} / \mathrm{s}$. Inset: trajectory of the center of mass of rotor optically trapped with a red laser (red) and free to diffuse (blue) (see main text and Methods). F) Migration of rotors in light gradient, for 5 different experiments. The rotors first exhibit a diffusive motion in homogeneous light, (black curves, $15 \mathrm{~s}$ for each experiment) but migrate towards the bright spot (colored curves) of a focused laser light (circle at the center). Typical time for migration is $10-30 \mathrm{~s}$. All scale bars are $5 \mu \mathrm{m}$.

tion of the experiment ( $>20 \mathrm{~min}$ ). The handedness is random, and we measure $49.85 \%$ clockwise and $50.15 \%$ of counter clockwise for 1017 rotors.

We quantify and analyze the angular dynamics of the rotors. Assuming a $\delta$ correlated gaussian noise $\zeta(t)$, the phase $\Phi$ follows the Langevin equation ${ }^{72,73}$ :

$$
\frac{\mathrm{d} \Phi(t)}{\mathrm{d} t}=\omega+\sqrt{2 D_{\Phi}} \zeta(t)
$$

with $\omega$ the mean angular speed, and $D_{\Phi}$ the rotational diffusion coefficient of the rotors. From this, we compute the angular fluctuations $\Delta \Phi^{\prime 2}=2 D_{\Phi} t$ with $\Phi^{\prime}=(\Phi-\omega t)$ by analyzing the angular dynamics of 12 individual rotors with the speed $\omega$ extracted as the mean value of the angular speed. The ensemble average is linear in time, as shown in Fig.3D and gives an amplitude for the angular noise, $D_{\Phi}=0.03 \pm 0.01 \mathrm{rad}^{2} / \mathrm{s}$. It compares with $\frac{3}{4} \cdot \frac{D_{R}}{R^{2}}$, where $D_{R}$ is the translational diffusion measured for the center of mass of the rotors, which stresses the common origin of active noise for the translational and angular fluctuations. Linking the translational and angular active noise as given by the Stokes Einstein relation in an equilibrium system, we find that the temperature of the active bath reaches $T \approx 1700 \mathrm{~K}^{59}$.

We further use the blue laser to generate light gradients and manipulate rotors. Initially, we assemble rotors that spin un- der uniform light. We then superimpose a light gradient using a blue laser. Swimmers constituting the rotors reorient by phototaxis without breaking the microgear. A slow migration, $V \sim 1$ $\mu \mathrm{m} / \mathrm{s}$, towards the laser spot follows. The rotor reshapes but does not break showing their robustness under light manipulation (see Fig.3F and supplementary movie S5). After extinction of the light gradient, the peripheral swimmers collectively reorient and the structure spins. A summary of the properties of the individual rotors is presented in Fig.3. We now focus our interest in the quantification of the effective interactions induced by the presence of the rotors.

\section{Anisotropic and dynamic diffusiophoretic interactions}

In this section, we quantify the strength and shape of the diffusiophoretic interactions originating from the chemical activity of the photocatalytic components of the rotors and compare it with our experimental measures. We use HILO microscopy to image and quantify the effective interaction created by a rotor on neighboring particles as measurements with conventional fluorescence microscopy techniques are overwhelmed by background noise and do not permit to measure accurately anisotropic interactions in the rotating frame of the rotor. We use small tracers, size $\sim 20$ 

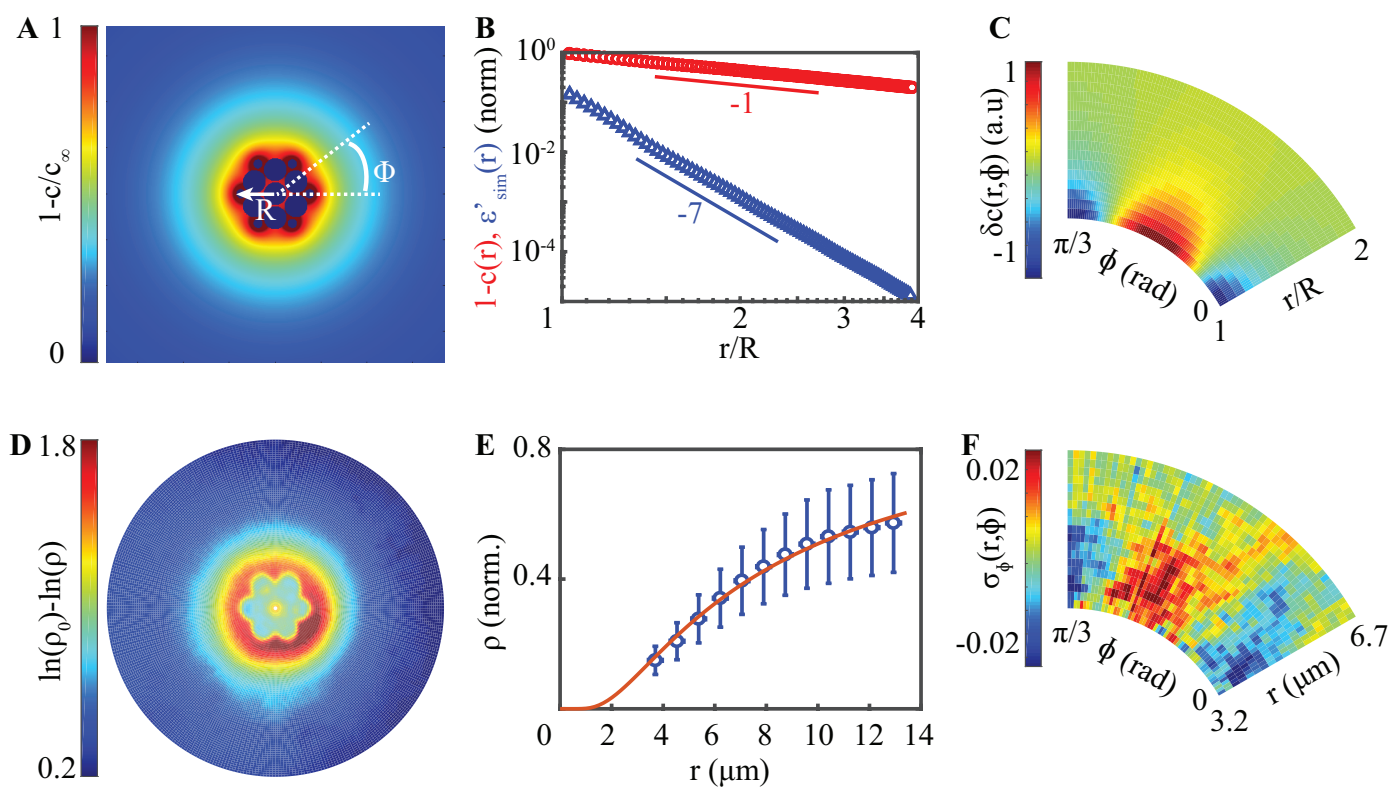

Fig. 4 Anisotropic and diffusiophoretic interactions. A), B), C) are simulations and D), E), F) are experiments. A) Numerical mapping of the (normalized) concentration field around a rotor of radius $R$, situated above an impermeable interface. At fixed distance, the concentration profile is periodically modulated by the presence of the haematite. B) Evolution of the radial concentration profile (after azimuthal average, red circles), following $\mathrm{a} \propto 1 / r$ decay. The azimuthal profile follows a sinusoidal variation in the interval $[0 ; \pi / 3]$ with decaying amplitude $\varepsilon_{\text {sim }}^{\prime} \propto 1 / r^{7}$ (blue triangles, see main text). C) Mapping of the azimuthal variations after subtracting the radial dependency (see main text). D) Experimental observation of the interaction profile between a rotor and $20 \mathrm{~nm}$ fluorescent beads using HILO microscopy, in the reference frame of the rotor. The data closely resemble A). E) The radial density profile of the fluorescent beads (blue circles) follows eq.(2) (red line), with $\alpha=120 \mu \mathrm{m}^{3} / \mathrm{s}$. F) Experimental mapping $\sigma_{\phi}$ of the azimuthal interactions after removal of the radial contribution (see main text). A periodic modulation is visible, with the higher repulsion near the haematite.

$\mathrm{nm}$, whose fast diffusive motion allows measuring the phoretic repulsion. Following, we perform a statistical analysis of the density of beads, averaged in the vertical direction as a result of the quick equilibration of the tracers, and extract the interaction potential. It differs from velocimetry experiments, previously performed to image the flow lines around microswimmers $60,74,75$, in that it allows us to access vanishing velocities of tracers, down to $0.1 \mu \mathrm{m} / \mathrm{s}$, for which diffusion is important in the so-called low Péclet regime $P e \sim V^{2} / R D_{\mathrm{c}} \leq 1$. Besides, thanks to the quick diffusion of chemicals compared to the rotation speed of the rotors, $\omega R^{2} / D<<1$, we can assume the equilibration of the chemicals as instantaneous and their concentration field steady in the rotating frame of the rotor.

\subsection{Analytical solution}

We first derive an analytical expression considering a simplified model. We model the rotor by a sphere, with a surface activity $v\left(r=\mathbf{R}_{\mathbf{s}}, \theta, \phi\right)$ in spherical coordinates. The modulation of surface activity $v$ reflects the positioning of the haematite on the periphery of the rotor. The concentration $c$ of $\mathrm{H}_{2} \mathrm{O}_{2}$ follows ${ }^{50}$ :

$$
\begin{aligned}
& D \nabla^{2} c=0 \\
& -D \mathbf{n} \cdot \nabla c\left(\mathbf{R}_{\mathbf{s}}\right)=v\left(\mathbf{R}_{\mathbf{s}}\right)
\end{aligned}
$$

Note that the concentration of chemical products follows the same set of equations eq.(9). We expand the concentration on a basis of spherical harmonics $Y_{l}^{m}(\theta, \phi)$. For a surface activity of the form $v(\theta, \phi)=\sum_{l=0}^{\infty} \sum_{m=-l}^{m=+l} v_{l m} Y_{l}^{m}(\theta, \phi)$, we get from (9):

$$
c(r, \theta, \phi)=c_{\infty}+\frac{R}{D} \sum_{l=0}^{\infty} \sum_{m=-l}^{m=+l} \frac{v_{l m}}{l+1}\left(\frac{R}{r}\right)^{l+1} Y_{l}^{m}(\theta, \phi)
$$

The decay of the concentration is given by the multipolar order of the surface activity. We prescribe a surface activity $v(\theta, \phi)=$ $v_{0}\left(1-\sin ^{6} \theta \cos 6 \phi\right)$, where the hexapolar symmetry of the rotor is given by $\cos 6 \phi$ term and $\sin ^{6} \theta$ confines the activity to the plane of the rotor. It conveniently gives $v \propto Y_{6}^{6}(\theta, \phi)+Y_{6}^{-6}(\theta, \phi)$, such that only $l=0,6$ remain in the series expansion of eq.(10):

$$
c(r, \theta, \phi)=c_{\infty}-\frac{v_{0} R^{2}}{D r}-\frac{v_{0} R^{8}}{7 D r^{7}} \cos 6 \phi \sin ^{6} \theta
$$

which we write

$$
c(r, \theta, \phi)=c_{\infty}-\alpha^{\prime}(r)-\varepsilon^{\prime}(r) \cos 6 \phi \sin ^{6} \theta
$$

with $\alpha^{\prime}=v_{0} R^{2} / D r$ and $\varepsilon^{\prime}=v_{0} R^{8} / 7 D r^{7}$. The parameter $\alpha^{\prime}$ represents the amplitude of the long-range radial diffusiophoretic interaction, while the strength of the azimuthal interaction is given by $\varepsilon^{\prime}$. They can be experimentally extracted by measuring the density profile of fluorescent beads around a rotor, and according to the model, their ratio is $\varepsilon^{\prime} r^{6} / \alpha^{\prime} R^{6}=1 / 7$. Using particles to probe the diffusiophoretic interactions, as we will do in the experiment (see sec. 5.3), the conservation of number of probes follows eq. (1) at $(r, \theta, \phi)$. At steady state, $\mathbf{J}=0$, it leads to the 
following expression for the density $\rho$ of probe particles:

$$
\rho(r, \phi, \theta)=\rho_{0} \exp \left(-\alpha^{\prime}-\varepsilon^{\prime} \cos 6 \phi \sin ^{6} \theta\right)
$$

For later convenience, we define:

$$
\sigma_{\phi}(r, \phi)=\ln \rho(r, \phi)-\langle\ln \rho(r, \phi)\rangle_{\phi}=\varepsilon^{\prime} \cos 6 \phi
$$

for $\theta=\pi / 2$ (XY plane of the rotor). This quantity will serve to experimentally evaluate $\varepsilon^{\prime}(r) \propto 1 / r^{7}$, as we shall see.

\subsection{Numerical simulations}

We numerically solve the steady diffusion equation of the $\mathrm{H}_{2} \mathrm{O}_{2}$ concentration $\Delta c=0$ around a rotor in 3D (COMSOL Multiphysics 5.3). We model the rotors as 7 passive and impermeable spheres (one central and six peripheral) of radius $R_{c}$, decorated at the periphery by 6 chemically active core-shell spheres of radius $R_{c} / 2$, plus one at the top of the central swimmer. The fuel consumption is imposed by a constant reaction rate occurring in a thin shell of $R_{c} / 10$. The cores of the active particles and the passive spheres are set as impermeable, with no-flux boundary condition. We position the rotor at distance $R_{c} / 10$ above an (infinite) impermeable wall constituted by the bottom surface of the capillary in the experiment. The concentration is set at 1 at infinity in upper space using Infinite Element Domain (COMSOL) to simulate infinite boundary conditions in a spherical coordinate system.

We first extract the concentration profile in the radial direction after azimuthal averaging in the $\mathrm{XY}$ plane, and measure a $1 / r$ decay (see Fig.4A-B). We then extract the azimuthal dependence of the concentration and calculate $\delta c(r, \phi)=c(r, \theta=$ $\pi / 2, \phi)-\langle c(r, \theta=\pi / 2, \phi)\rangle_{\phi}$. For each radial distance $r, \delta c(\phi)$ curve show a cosine shape (Fig.4C), from which we extract the amplitude $\varepsilon_{\mathrm{sim}}^{\prime}(r)=\left[\delta c_{\max }-\delta c_{\min }\right] / 2$. The evolution of $\varepsilon_{\mathrm{sim}}^{\prime}(r)$ is shown in Fig.4B, where it decays as $1 / r^{7}$, in agreement with the analytical model and eq.(11). In particular, we extract the ratio $\varepsilon^{\prime}(r) r^{6} / \alpha^{\prime} R^{6}=0.18 \pm 0.04$ for $1<r / R<4$. This ratio is in good agreement with the analytical model, which predicts $\varepsilon^{\prime} r^{6} / \alpha^{\prime} R^{6} \approx 0.14$

\subsection{Optical imaging of the diffusiophoretic interactions}

We aim at comparing our predictions for the diffusiophoretic interactions with experimental measurements. We visualize the effective interaction around a single rotor by dispersing $20 \mathrm{~nm}$ nile red fluorescent beads in the solution that exhibit diffusiophoretic motion in chemical gradients (Fig.4D and setup description in the Methods section). We first form a rotor and suppress the random motion of its center of mass (see Methods) by optically trapping its center with a red laser. We lower the intensity of the illumination to slow down the dynamics of the rotor and permit equilibration of the fluorescent beads, necessary to image the -steadydensity profile in the reference frame of the rotor. We use a homebuilt HILO microscopy setup (see Methods) to visualize a slice of a few $\mu \mathrm{m}$ above the substrate and suppress the bulk background fluorescence (see Methods). Care was taken to carefully align the trapping laser with the center of the HILO illumination, where the depth of the excitation field is minimal. We then excite the beads and record their fluorescence during $1 \mathrm{~s}$ time intervals, imposing dark periods of $4 \mathrm{~s}$ between successive excitation cycles, preventing photo-bleaching of the area around the rotor. The sequence is repeated for 10 cycles, or until the rotor breaks. We record the background fluorescence in independent experiments under the same excitation conditions and correct accordingly. We analyze

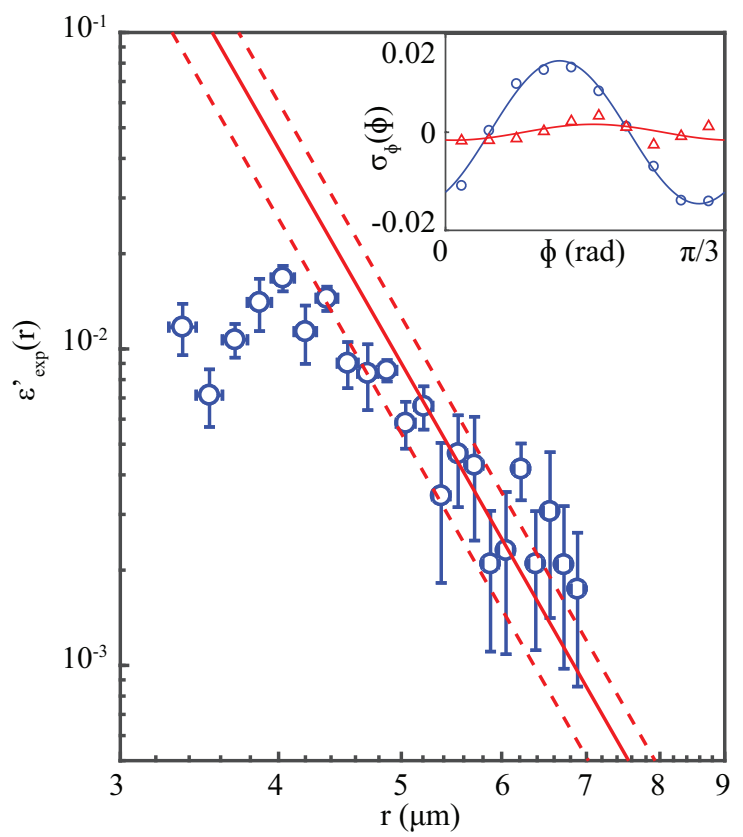

Fig. 5 Experimental determination of the azimuthal interaction. Evolution of the amplitude of oscillations shown in Fig.4F, as a function of the distance decaying as $\propto 1 / r^{7}$. Red solid and dashed lines are the mean value and its extrema, respectively, as predicted by the numerical simulation in the absence of adjustable parameter (see main text). It shows an excellent agreement with the experimental data. Inset: Profiles of Fig.4F, at distances of 4 (blue circles) and $6 \mu \mathrm{m}$ (red triangles) from the center of the rotor. Solid lines are fits using a sinusoidal function. It confirms diffusiophoresis as the source of interaction.

the movies by measuring the instantaneous fluorescence profile around a rotor in the rotating frame $(r, \phi)$. Averaging over all the frames, we obtain the intensity profile (Fig.4D), further averaged over $\phi \in[0 ; \pi / 3]$ by rotor symmetry.

In the far field, the concentration of fluorescent particles follows $\rho=\rho_{0} e^{-\alpha /\left(D_{c} r\right)}$ as prescribed in eq. (2) for a sink of $\mathrm{H}_{2} \mathrm{O}_{2}, c \propto 1 / r$ (Fig.4E). We extract the amplitude of the radial phoretic repulsion $\alpha=120 \pm 30 \mu \mathrm{m}^{3} / \mathrm{s}$, in line with the results from the experiments realized with haematite alone and the same fluorescent beads (section 2). In the near field, $1<\mathrm{r} / \mathrm{R}<2$, the concentration field is hexapolar and we investigate the azimuthal gradient of concentration by computing the experimental values for $\sigma_{\phi}=\ln \rho(r, \phi)-\langle\ln \rho(r, \phi)\rangle_{\phi}$. We observe a small modulation of the amplitude in the interval $[0 ; \pi / 3]$, for distances $r \sim 3.2-6.7 \mu \mathrm{m}$ (Fig.4F), with the maximal repulsion near the haematite. Guided by the analytical model [eq.(14)], we adjust the data with the function $\varepsilon_{\exp }^{\prime} \cos \left(6 \phi+\phi_{0}\right)$ using $\varepsilon_{\exp }^{\prime}$ and $\phi_{0}$ as fitting parameters and repeat the procedure for different distance $r$ to the center of the rotor, giving $\varepsilon_{\exp }^{\prime} \propto 1 / r^{7}$ (Fig.5), as predicted by eq.(13). We further compare the experimental value with the estimate from 
the numerical simulations using $\varepsilon_{\text {sim }}^{\prime}(r) r^{6} / \alpha^{\prime} R^{6}=0.18 \pm 0.04$ (section 5.2) and $\alpha=120 \mu \mathrm{m}^{3} / \mathrm{s}$, showing an excellent agreement without adjustable parameters (Fig.5). Further, those interactions can be used to synchronize multiple rotors and in fact, the results hereby described with probe particles agree with the phoretic coupling parameter measured in ref. ${ }^{76}$ for interacting rotors. The quantitative agreement between the experimental and the diffusiophoretic model stresses that hydrodynamics flows do not seem to have a dominant effect in the present experiment, as confirmed by the observed direction of co-rotation of co-rotating pairs inconsistent with a simple hydrodynamic advection ${ }^{76}$. It highlights diffusiophoresis as a powerful tool to shape and program interactions with quantitative control.

\section{Conclusion}

We demonstrated the assembly of hierarchical structures from a single type of dissipative building block. To this end, we used diffusiophoretic phenomena to guide the self-assembly and encode the dynamics. Starting from a photoactive material, the haematite, we designed phototactic microswimmers that migrate toward high intensity of light. They spontaneously assemble into self-spinning microgears, that rotate and sustain in the absence of any feedback from the operator. We introduced an optical method - HILO microscopy - as a novel tool to characterize phoretic interactions. The method is generic and can be implemented to image phoretic interactions around (self)-phoretic swimmers. It reveals anisotropic, periodic near field modulations of the chemical cloud around rotors, which amplitude compares with both numerical simulations and a simple analytical model. This rational design of machines from machines shows the opportunities offered by phoretic phenomena to control dissipative self-assembly, and paves the way for later studies of large assemblies of spinning structures.

\section{Materials and Methods}

\subsection{Sample preparation}

The samples are prepared at low particle density $\Phi_{s} \sim 10^{-3}$ part $/ \mu \mathrm{m}^{2}$. Particles are diluted in a $6 \%$ solution of hydrogen peroxide $\mathrm{H}_{2} \mathrm{O}_{2}$ (Fisher Scientific H325-500) in deionized water (Milli-Q, resistivity $18.2 \mathrm{M} \Omega$ ). The final solution has a $\mathrm{pH}$ of $\sim 6-7$. The solution is then injected in a small rectangular capillary (VitroCom 3520-050), and sealed with capillary wax (Hampton Research HR4-328). All the capillaries are previously subjected to plasma-cleaning (Harrick Plasma PDC-001) and rinsed thoroughly with deionized water. As the particles are non-buoyant, they sediment near the bottom surface of the capillary, and observation is made in this plane on an home-built microscope.

Synthesis of the particles is described in details in the supplementary information of ref. ${ }^{76}$.

\subsection{Measurements of intensities}

- To extract the intensity profile of the blue laser in sections 2 and 3, we deposit fluorescent beads (Lifetechnologies F8888, diameter $20 \mathrm{~nm}$ ) on the surface of a capillary, and fill it with water to obtain the fluorescence intensity pattern along the radial direction after azimuthal averaging.

- The maximum power from LED2 arriving on the sample is measured to be of the order of $\sim 10 \mathrm{~mW}$, on an area of roughly $150 \times 150 \mu \mathrm{m}^{2}$. This corresponds to an intensity $I_{\max }$ of $\sim 500$ $\mathrm{nW} / \mu \mathrm{m}^{2}$. We find $I_{\mathrm{sat}} / I_{\max }=9 \%$ and thus $I_{\mathrm{sat}} \sim 50 \mathrm{nW} / \mu \mathrm{m}^{2}$.

- For the blue laser, the shape of the intensity profile in Fig.1C is used to compute the peak intensity for a $1 \mathrm{~mW}$ power, and gives $I_{\text {peak }}=2000 \mathrm{nW} / \mu \mathrm{m}^{2}$. From the experiments, we extract $I_{\text {sat }}=75$ $\mathrm{nW} / \mu \mathrm{m}^{2}$.

\subsection{Optical setup for rotor creation}

The custom optical setup is represented on Fig.6A. The sample is observed with optical microscopy on an inverted microscope and with bright field transmitted illumination (LED1). A LED is set up in the blue range (LED2, $\lambda=425-500 \mathrm{~nm}$, Lumencore SOLA 6LCR-SC) and uniformly illuminates the sample on a large area (up to $300 \times 300 \mu \mathrm{m}^{2}$ ) to trigger the photo-catalysis of haematite, after being reflected on an appropriate dichroic mirror. A laser diode source L1 ( $\lambda=404 \mathrm{~nm}$, Thorlabs L404P400M) is superimposed to the homogeneous excitation profile. It is focused in a multimode (MM) optical fiber F1 (Thorlabs M42L02), re-collimated at the output, and reflected on a manually adjustable mirror (A.M). The beam is then reflected on a dichroic mirror (Thorlabs DMLP425R) before being focused on the sample through a high Numerical Aperture (NA) oil-immersion objective (Nikon $60 \mathrm{x}, \mathrm{NA}=1.4$ ). The adjustable mirror allows steering the laser spot along the surface of the sample and selecting the location for rotor formation. Furthermore, an electronic shutter (Thorlabs SHB1T) on the optical path enables switching ON and OFF the laser spot. The sample is mounted on a manual micrometric stage (Nikon Ti-SR). We observe the image of the sample through the 60x objective on a monochrome CCD camera (Edmund Optics EO-1312M), with a set of appropriate optical filters. Acquisitions are performed at typical rates of $20-100 \mathrm{fps}$.

\subsection{Optical setup for HILO microscopy}

\subsubsection{HILO microscopy}

We modified the optical setup presented in section 7 to analyze the phoretic repulsion of fluorescent beads around a haematite and a rotor (Fig.6B). We used Highly Inclined and Laminated Optical sheet microscopy (HILO, also called near-TIRF) to visualize the fluorescence in a thin slice $(\sim$ few $\mu \mathrm{m})$ above the surface ${ }^{55-57}$. The setup is similar to a Total Internal Reflection Fluorescence Microscopy setup. In HILO microscopy, the beam arrives at an angle slightly smaller than the critical angle, such that the beam is highly inclined, and not totally reflected. This results in the illumination of a thin slice with a width of typically a few microns $^{55}$ (Fig.6C-D) and therefore much lower background fluorescence compared to epifluorescence microscopy, as exemplified in Fig.6D. 

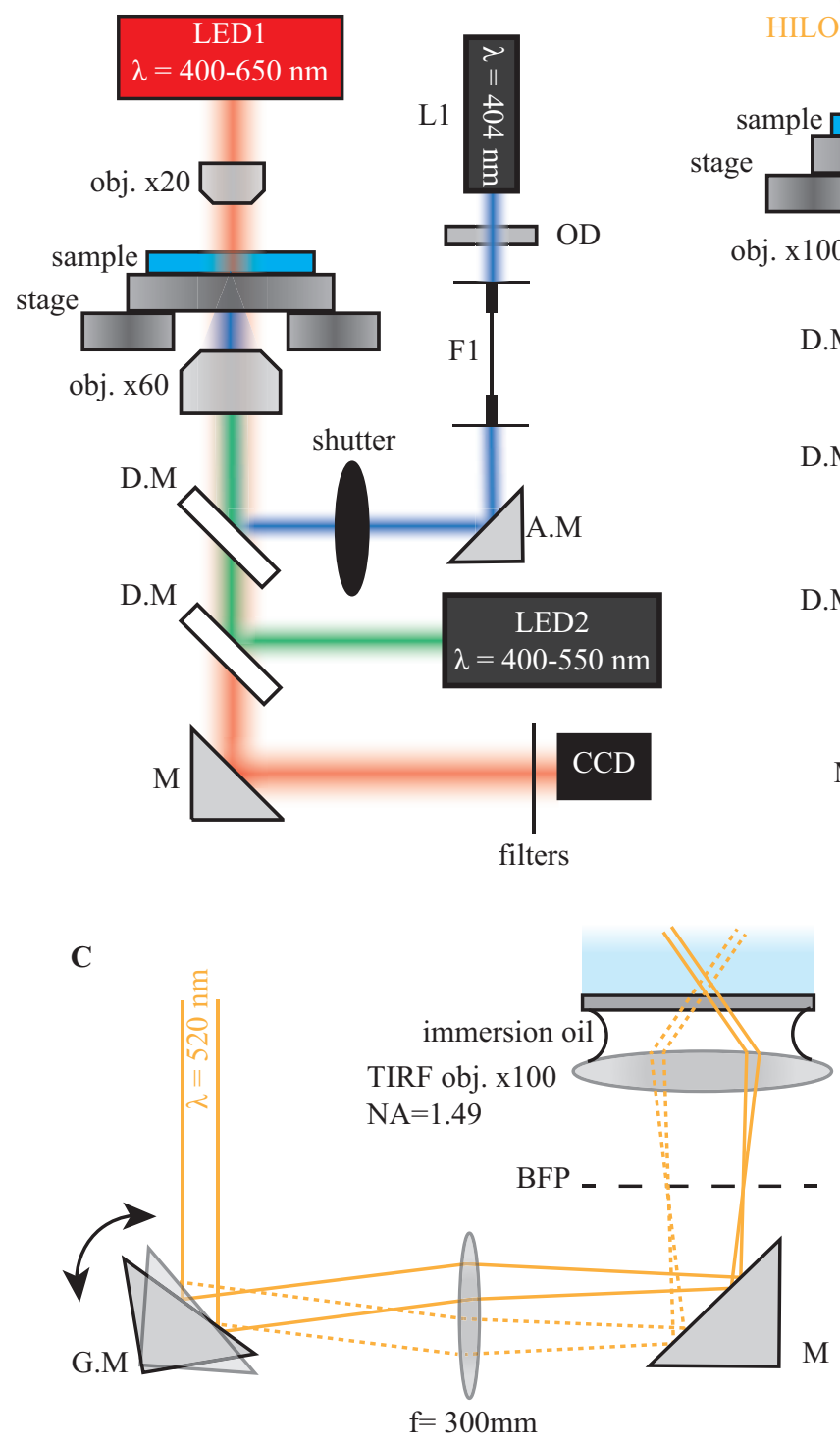

B

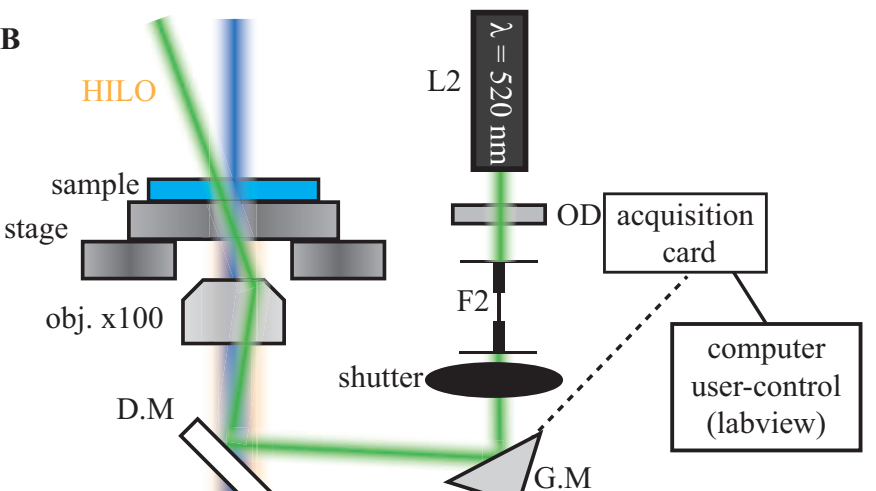

D.M

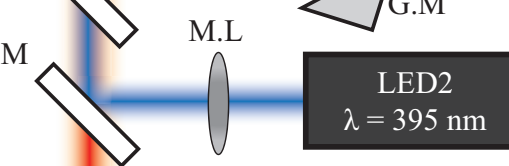

D.M

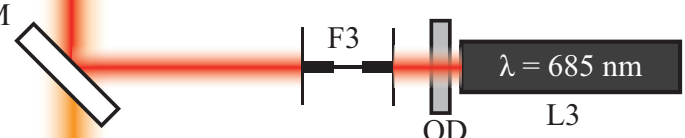

M

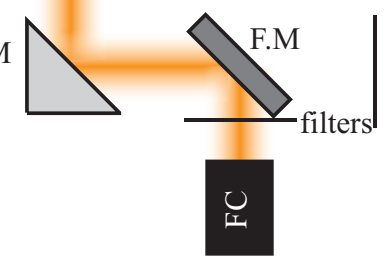

CCD

D
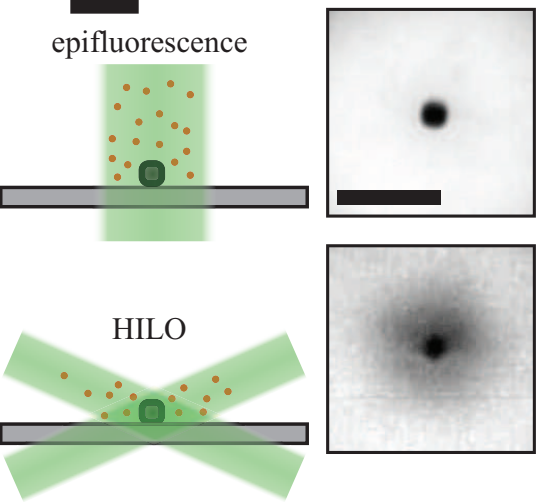

Fig. 6 Experimental setups. See main text for full description. A) Experimental setup used for rotor creation. B) Highly Inclined Laminated Optical sheets (HILO) microscopy setup used for imaging the concentration profile around a rotor. C) Detailed scheme showing the light path for HILO microscopy. The laser is focused at the back focal plane of a high-NA objective. Shifting the position of the laser with respect to the center of the entrance pupil results in inclined, output light beams. D) Top:In standard epifluorescence microscopy, background fluorescence is high because of strong illumination of the planes above the focal plane. Bottom:In HILO microscopy, only a small slice ( $\sim$ few microns) above the focal plane is shined, lowering the background fluorescence. The images on the right show the fluorescence profile of beads around a haematite in epifluorescence (top), compared to HILO (bottom). Scale bar is $5 \mu \mathrm{m}$. The symbols used are : L1, L2, L3 : Laser sources; D.M : Dichroic Mirror; G.M : 2-axis Galvanometric Mirror; F1: Multimode Fiber; F2, F3: Single Mode fibers; OD: Optical Densities; F.M : Flip Mirror; A.M : Adjustable Mirror M.L : Movable Lens; F.C : Fluorescence Camera; $C C D$ : Charged-Coupled-Device camera; $M$ : Mirror. For better clarity, we do not represent optical lenses.

\subsubsection{Optical setup}

We used nile red fluorescent beads (F8784, ThermoFisher Scientific) to image the concentration profile, so that excitation at $\sim 520 \mathrm{~nm}$ for fluorescence at $\sim 575 \mathrm{~nm}$ can be done without activating the haematite, weakly absorbing at this wavelength. The blue laser light is replaced by a $520 \mathrm{~nm}$ laser light source L2 (PL520, Thorlabs, $50 \mathrm{~mW}$ ) and focused in a single mode fiber F2 (SM, Thorlabs, P1-460B-FC-2) so that the resulting output beam mode is gaussian. The beam is collimated again, and sent in a 2D galvanometric mirror system (G.M, Thorlabs GVS212). Us- ing a long-focal achromatic lens $(300 \mathrm{~mm})$, the beam is focused at the back focal plane of a TIRF objective with high numerical aperture (apo-TIRF, x100, NA=1.49, NIKON). The output beam from the objective is collimated, and its angle with the surface of the sample is adjusted by controlling the orientation of the galvanometric mirror, addressed in real time through an acquisition card (NI USB-6343) with a home-made user-controlled interface developed under Labview 2017. In particular, we impose a high frequency $(100 \mathrm{~Hz})$ oscillatory motion of the mirror to scan over $2 \pi$ the entrance pupil of the objective with the laser beam. This 
technique guarantees an isotropic illumination profile.

We form the rotors by changing the wide-field excitation pattern of LED2 using a movable lens (ML). The swimmers migrate toward the high intensity area, so that reducing the size of the light spot increases the density and eventually triggers the formation of the rotor. Adjusting the position of the lens back to its original position disperses the swimmers again in a homogeneous intensity profile and keeps the rotor active. Finally, a red laser L3 ( $\lambda=685 \mathrm{~nm}$, Thorlabs, HL6750MG, $50 \mathrm{~mW}$ ) is added to the setup to trap the rotors. The beam is focused in a single mode fiber F3 (SM, P1-630A-FC-2), recollimated at the output, and reflected on a shortpass dichroic mirror (Thorlabs, DMSP650R). The position of the beam is carefuly adjusted and centered on the HILO excitation pattern. We observe the fluorescence of the beads on a fluorescence camera (Photometrics Scientific, Coolsnap HQ2), with a resolution of $0.168 \mu \mathrm{m} / \mathrm{pixel}$, and with an integration time of $50 \mathrm{~ms}$. A flip mirror (F.M) is inserted on the detection path to switch between viewing of the fluorescence or the bright field image on the CCD camera, using sets of appropriate spectral filters for each camera. The overall setup allows us to form and trap a rotor, and to observe its near-field concentration cloud through HILO microscopy.

\section{Conflicts of interest}

The authors declare no conflicts of interests

\section{Acknowledgements}

The authors gratefully acknowledge Stefano Sacanna and Mena Youssef from New York University for supplying the colloidal particles. This material is based on work supported by the National Science Foundation under Grant No. DMR-1554724. J.P. thanks the Sloan Foundation for support through grant FG-2017-9392.

\section{References}

1 L. Cademartiri and K. J. M. Bishop, Nature Materials, 2014, 14, 2.

2 K. F. Wagenbauer, C. Sigl and H. Dietz, Nature, 2017, 552, 78.

3 M. Y. Ben Zion, X. He, C. C. Maass, R. Sha, N. C. Seeman and P. M. Chaikin, Science, 2017, 358, 633-636.

4 S. Sacanna, W. T. M. Irvine, P. M. Chaikin and D. J. Pine, Nature, 2010, 464, 575.

5 S. C. Glotzer and M. J. Solomon, Nature Materials, 2007, 6, 557.

6 Q. Chen, S. C. Bae and S. Granick, Nature, 2011, 469, 381.

7 B. Bharti and O. D. Velev, Langmuir, 2015, 31, 7897-7908.

8 T. O. Tasci, P. S. Herson, K. B. Neeves and D. W. M. Marr, Nature Comm, 2016, 7, 10225.

9 A. Snezhko and I. S. Aranson, Nature Materials, 2011, 10, 698.

10 R. M. Erb, H. S. Son, B. Samanta, V. M. Rotello and B. B. Yellen, Nature, 2009, 457, 999.

11 D. G. Grier, Nature, 2003, 424, 810.

12 T. Li, J. Li, K. I. Morozov, Z. Wu, T. Xu, I. Rozen, A. M. Leshansky, L. Li and J. Wang, Nano Lett., 2017, 17, 5092-5098.
13 K. Han, C. W. Shields, N. M. Diwakar, B. Bharti, G. P. López and O. D. Velev, Science Advances, 2017, 3, year.

14 M. C. Marchetti, J. F. Joanny, S. Ramaswamy, T. B. Liverpool, J. Prost, M. Rao and R. A. Simha, Rev. Mod. Phys., 2013, 85, 1143-1189.

15 W. Wang, W. Duan, S. Ahmed, A. Sen and T. E. Mallouk, Accounts of Chemical Research, 2015, 48, 1938-1946.

16 B. A. Grzybowski, H. A. Stone and G. M. Whitesides, Nature, 2000, 405, 1033-1036.

17 B. A. Grzybowski and G. M. Whitesides, Science, 2002, 296, 718-721.

18 M. Youssef, T. Hueckel, G.-R. Yi and S. Sacanna, Nature Comm, 2016, 7, 12216.

19 S. Sacanna, M. Korpics, K. Rodriguez, L. Colón-Meléndez, S.H. Kim, D. J. Pine and G.-R. Yi, Nature Comm, 2013, 4, 1688.

20 A. Aubret, S. Ramananarivo and J. Palacci, Current Opinion in Colloid \& Interface Science, 2017, 30, 81 - 89.

21 K. K. Dey, F. Wong, A. Altemose and A. Sen, Current Opinion in Colloid \& Interface Science, 2016, 21, 4-13.

22 A. Zottl and H. Stark, Journal of Physics: Condensed Matter, 2016, 28, 253001.

23 J. Yan, M. Han, J. Zhang, C. Xu, E. Luijten and S. Granick, Nat Mater, 2016, 15, 1095-1099.

24 J. Yan, M. Bloom, S. C. Bae, E. Luijten and S. Granick, Nature, 2012, 491, 578-581.

25 M. Fialkowski, K. J. M. Bishop, R. Klajn, S. K. Smoukov, C. J. Campbell and B. A. Grzybowski, J. Phys. Chem. B, 2006, 110, 2482-2496.

26 J. L. England, Nature Nanotechnology, 2015, 10, 919.

27 J. Zhang, J. Yan and S. Granick, Angew. Chem. Int. Ed., 2016, 55, 5166-5169.

28 F. Ma, S. Wang, D. T. Wu and N. Wu, Proceedings of the National Academy of Sciences, 2015, 112, 6307-6312.

29 S. Das, A. Garg, A. I. Campbell, J. Howse, A. Sen, D. Velegol, R. Golestanian and S. J. Ebbens, Nature Comm, 2015, 6, 8999.

30 J. Simmchen, J. Katuri, W. E. Uspal, M. N. Popescu, M. Tasinkevych and S. Sánchez, Nature Comm, 2016, 7, 10598.

31 J. Katuri, D. Caballero, R. Voituriez, J. Samitier and S. Sanchez, ACS Nano, 2018.

32 A. T. Brown, I. D. Vladescu, A. Dawson, T. Vissers, J. SchwarzLinek, J. S. Lintuvuori and W. C. K. Poon, Soft Matter, 2016, 12, 131-140.

33 D. Takagi, J. Palacci, A. B. Braunschweig, M. J. Shelley and J. Zhang, Soft Matter, 2014, 10, 1784-1789.

34 M. Claudio, S. Juliane, S. Filippo, K. Jaideep, D. Michele, D. A. Francesco, S. Samuel and D. L. Roberto, Small, 2016, 12, 446-451.

35 G. Vizsnyiczai, G. Frangipane, C. Maggi, F. Saglimbeni, S. Bianchi and R. Di Leonardo, Nature Comm, 2017, 8, 15974.

36 H. Eskandarloo, A. Kierulf and A. Abbaspourrad, Nanoscale, 2017, 9, 12218-12230.

37 M. Ibele, T. Mallouk and A. Sen, Angewandte Chemie International Edition, 2009, 48, 3308-3312. 
38 J. Palacci, S. Sacanna, A. P. Steinberg, D. J. Pine and P. M. Chaikin, Science, 2013, 339, 936-940.

39 W. Li, X. Wu, H. Qin, Z. Zhao and H. Liu, Advanced Functional Materials, 2016, 26, 3164-3171.

40 N. Chaturvedi, Y. Hong, A. Sen and D. Velegol, Langmuir, 2010, 26, 6308-6313.

41 C. Maggi, F. Saglimbeni, M. Dipalo, F. De Angelis and R. Di Leonardo, Nature Communications, 2015, 6, 7855.

42 B. Dai, J. Wang, Z. Xiong, X. Zhan, W. Dai, C.-C. Li, S.-P. Feng and J. Tang, Nat Nano, 2016, 11, 1087-1092.

43 J. Zheng, B. Dai, J. Wang, Z. Xiong, Y. Yang, J. Liu, X. Zhan, Z. Wan and J. Tang, Nature Communications, 2017, 8, 1438.

44 C. Lozano, B. ten Hagen, H. Löwen and C. Bechinger, Nature Communications, 2016, 7, 12828.

45 J. Palacci, S. Sacanna, S.-H. Kim, G.-R. Yi, D. J. Pine and P. M. Chaikin, Philosophical Transactions of the Royal Society of London A: Mathematical, Physical and Engineering Sciences, 2014, 372 , year.

46 J. Anderson, Annual Review of Fluid Mechanics, 1989, 21, 6199.

47 R. Golestanian, T. B. Liverpool and A. Ajdari, Phys. Rev. Lett., 2005, 94, 220801.

48 I. Buttinoni, G. Volpe, F. Kümmel, G. Volpe and C. Bechinger, Journal of Physics: Condensed Matter, 2012, 24, 284129.

49 J. R. Howse, R. A. L. Jones, A. J. Ryan, T. Gough, R. Vafabakhsh and R. Golestanian, Phys. Rev. Lett., 2007, 99, 048102.

50 R. Golestanian, T. B. Liverpool and A. Ajdari, New Journal of Physics, 2007, 9, 126.

51 J. Dervaux, M. Capellazzi Resta and P. Brunet, Nat Phys, 2017, 13, 306-312.

52 C. Chen, F. Mou, L. Xu, S. Wang, J. Guan, Z. Feng, Q. Wang, L. Kong, W. Li, J. Wang and Q. Zhang, Adv. Mater., 2017, 29, 1603374.

53 Q. He, z. Lin, t. si, Z. Wu, c. gao and X. Lin, Angewandte Chemie International Edition, 2017, n/a-n/a.

54 S.-S. Lin and M. D. Gurol, Environmental Science \& Technology, 1998, 32, 1417-1423.

55 M. Tokunaga, N. Imamoto and K. Sakata-Sogawa, Nature Methods, 2008, 5, 159.

56 S. Shashkova and M. Leake, Bioscience Reports, 2017, 37, BSR20170031.

57 Z. Liu, L. Lavis and E. Betzig, Molecular Cell, 2015, 58, 644659.

58 J. Palacci, S. Sacanna, A. Abramian, J. Barral, K. Hanson, A. Y. Grosberg, D. J. Pine and P. M. Chaikin, Sci Adv, 2015, 1, year.

59 J. Palacci, C. Cottin-Bizonne, C. Ybert and L. Bocquet, Phys. Rev. Lett., 2010, 105, 088304.

60 H. Moyses, J. Palacci, S. Sacanna and D. G. Grier, Soft Matter, 2016, 12, 6357-6364.

61 B. ten Hagen, R. Wittkowski, D. Takagi, F. Kümmel, C. Bechinger and H. Löwen, Journal of Physics: Condensed Matter, 2015, 27, 194110.

62 Y. Li, F. Marchesoni, T. Debnath and P. K. Ghosh, Phys. Rev. E,
2017, 96, 062138.

63 W. E. Uspal, M. N. Popescu, S. Dietrich and M. Tasinkevych, Soft Matter, 2015, 11, 434-438.

64 J. Elgeti and G. Gompper, The European Physical Journal Special Topics, 2016, 225, 2333-2352.

65 D. Lopez and E. Lauga, Physics of Fluids, 2014, 26, 071902.

66 J. Stenhammar, A. Tiribocchi, R. J. Allen, D. Marenduzzo and M. E. Cates, Phys. Rev. Lett., 2013, 111, 145702.

67 M. E. Cates and J. Tailleur, Annu. Rev. Condens. Matter Phys., 2015, 6, 219-244.

68 R. Di Leonardo, F. Ianni and G. Ruocco, Langmuir, 2009, 25, 4247-4250.

69 B. Liebchen, D. Marenduzzo and M. E. Cates, Phys. Rev. Lett., 2017, 118, 268001.

70 B. Liebchen, D. Marenduzzo, I. Pagonabarraga and M. E. Cates, Phys. Rev. Lett., 2015, 115, 258301.

71 B. Liebchen and H. Löwen, ArXiv e-prints, 2018.

72 M. S. Davies Wykes, J. Palacci, T. Adachi, L. Ristroph, X. Zhong, M. D. Ward, J. Zhang and M. J. Shelley, Soft Matter, 2016, 12, 4584-4589.

73 M. Rosenblum and A. Pikovsky, Contemporary Physics, 2003, 44, 401-416.

74 K. Drescher, J. Dunkel, L. H. Cisneros, S. Ganguly and R. E. Goldstein, Proceedings of the National Academy of Sciences, 2011, 108, 10940-10945.

75 A. I. Campbell, S. J. Ebbens, P. Illien and R. Golestanian, ArXiv e-prints, 2018.

76 A. Aubret, M. Youssef, S. Sacanna and J. Palacci, Nature Physics, 2018. 
Rotation of a self-assembled phoretic microgear Page 13 of 13 Soft Matter 